\title{
Efficacy and Safety of Anlotinib Combined with Liposomal Doxorubicin Followed by Anlotinib Maintenance in Metastatic Soft Tissue Sarcomas
}

This article was published in the following Dove Press journal: Cancer Management and Research

\author{
Zhiyong Liu' \\ Weitao Yao' \\ Yao Zhao ${ }^{2}$ \\ Oufei $\mathrm{Liu}^{3}$ \\ Peng Zhang' \\ Hong $\mathrm{Ge}^{4}$ \\ 'Department of Orthopedics, The \\ Affiliated Cancer Hospital of Zhengzhou \\ University and Henan Cancer Hospital, \\ Zhengzhou, Henan, People's Republic of \\ China; ${ }^{2}$ Department of Orthopedics, \\ The First Affiliated Hospital of \\ Zhengzhou University, Zhengzhou, \\ Henan, People's Republic of China; \\ ${ }^{3}$ Department of Oncology, The Fifth \\ Affiliated Hospital of Zhengzhou \\ University, Zhengzhou, Henan, People's \\ Republic of China; ${ }^{4}$ Department of \\ Radiation Oncology, The Affiliated \\ Cancer Hospital of Zhengzhou \\ University and Henan Cancer Hospital, \\ Zhengzhou, Henan, People's Republic of \\ China
}

Purpose: Anlotinib is a novel tyrosine kinase inhibitor with promising anti-tumor activity in patients with advanced soft tissue sarcomas (STS) in China. Liposomal doxorubicin monotherapy showed an encouraging effect on this disease. The aim of this study was to evaluate the efficacy and safety of anlotinib combined with liposomal doxorubicin followed by anlotinib maintenance in patients with metastatic STS.

Patients and Methods: This is a multicenter, retrospective, observational study. We reviewed 27 patients with metastatic STS from July 2018 to December 2019, who were treated with anlotinib combined with liposomal doxorubicin followed by anlotinib maintenance in the absence of the tumor progression or intolerable adverse events (AEs).

Results: Of the 27 patients included, 2 patients had complete response (CR), 9 patients obtained partial response (PR), 11 patients achieved stable disease (SD). The objective response rate was $40.7 \%$, the disease control rate was $81.5 \%$, and the median progressionfree survival (PFS) was 7 months (95\% CI, 5.3-8.1 months). The progression-free rate (PFR) at 3 and 6 months was $81.5 \%$ and $59.3 \%$, respectively. Most AEs were mild and acceptable. The most frequent grade 3/4 AEs were leukopenia (33.3\%), febrile neutropenia (7.4\%), and anemia $(7.4 \%)$. No deaths related to the treatment were reported.

Conclusion: This study shows that anlotinib combined with liposomal doxorubicin followed by anlotinib maintenance is effective in patients with metastatic STS, and most AEs of this combined therapy are mild and acceptable. Further investigation on its efficacy is warranted.

Keywords: anlotinib, liposomal doxorubicin, soft tissue sarcomas, efficacy, safety

\section{Introduction}

Soft tissue sarcomas (STS) represent a rare heterogeneous group of malignant tumors originating from mesenchymal tissue, with more than 50 histological subtypes. ${ }^{1}$ The mainstay treatments for this disease include surgical resection, radiotherapy, and chemotherapy, approximately half of the patients will eventually develop into locally unresectable or metastatic STS. Doxorubicin is currently the standard first-line regimen for most patients with advanced STS. Outcomes for those patients are poor, with an objective response rate (ORR) of about 14\%, median progressive-free survival (PFS) of about 5 months and overall survival (OS) of 12 to 16 months. ${ }^{2,3}$ Various cytotoxic drugs are being used as second-line treatment after failure of doxorubicin-based therapy, among these drugs is docetaxel plus gemcitabine as one of the most widely used therapy, with
Correspondence: Hong Ge

Email doctorgehong@163.com
Cancer Management and Research 2021:13 1009-1016

1009

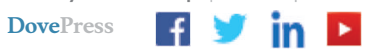

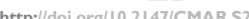


modest activity and significant hematological toxicity. ${ }^{4}$ Other more commonly used for specific tumors are highdose ifosfamide for synovial sarcoma, ${ }^{5}$ paclitaxel for angiosarcoma, ${ }^{6}$ and trabectedin for liposarcoma/ leiomyosarcoma. ${ }^{7}$ As an encapsulation agent of doxorubicin, liposomal doxorubicin shows similar efficacy with conventional doxorubicin in patients with advanced STS as first-line or later-line therapy, with favorable toxicity. Additionally, it could be administrated to patients who had received $450 \mathrm{mg} / \mathrm{m}^{2}$ conventional doxorubicin. ${ }^{8,9}$ These suggest that liposomal doxorubicin may be suitable backbone for novel combination strategies in STS.

Increasing evidence showed antiangiogenic therapy was a strategy for the treatment of advanced STS. Anlotinib is a novel tyrosine kinase inhibitor (TKI) targeting vascular endothelial growth factor receptor 1-3 (VEGFR1-3), c-kit and platelet-derived growth factor (PDGF). In a Phase I trial, anlotinib has demonstrated its safety and promising antitumor potential against many types of tumor including renal clear cell cancer and STS. ${ }^{10}$ In a large multicenter Phase II trial of patients with refractory metastatic STS after doxorubicin-based chemotherapy, patients receiving anlotinib gained good antitumor activity in advanced STS, the ORR was $13 \%$ and the progression-free rate (PFR) at 12-week was $68 \%$, with median PFS of 5.6 months and median OS of 12 months. ${ }^{11}$ In a Phase IIb trial of 233 patients with metastatic STS after failure of standard chemotherapy, anlotinib yielded a prolonged PFS (6.3 months vs 1.5 months), a high great ORR (10.1\% vs $1.3 \%)$ and disease control rate (DCR) (55.7\% vs 22.7\%) compared with placebo. ${ }^{12}$ Based on above results, anlotinib has been approved by China Food and Drug Administration (CFDA) for advanced STS after failure of standard chemotherapy since 2019.

Targeted drugs involving multiple signal pathways related to tumorigenesis may lead to benefits of cytotoxic chemotherapy. Preclinical studies showed that anlotinib and cytotoxic chemotherapy may lead to a synergistic effect, suggesting these drugs may reduce chemotherapy resistance. ${ }^{13}$ Considering the modest efficacy and mild toxicity of anlotinib and liposomal doxorubicin for advanced STS, a cohort of patients with advanced STS were treated with anlotinib combined with liposomal doxorubicin followed anlotinib maintenance for survival benefits in three hospitals since 2019. In this study, it aimed to assess the efficacy and safety of anlotinib combined with liposomal doxorubicin followed by anlotinib maintenance monotherapy in patients with metastatic STS.

\section{Patients and Methods}

\section{Patients}

This is a multicenter retrospective study of patients with STS who were treated with anlotinib combined with liposomal doxorubicin followed by anlotinib monotherapy, and it was conducted between July 2018 and December 2019 in three hospitals: The Affiliated Cancer Hospital of Zhengzhou University, The Fifth Affiliated Hospital of Zhengzhou University, and The First Affiliated Hospital of Zhengzhou University. The eligibility criteria were as follows: (1) between 18 and 65 years of age; (2) histologically confirmed diagnosis of STS; (3) at least one measurable metastatic lesion according to the Response Evaluation Criteria in Solid Tumors (RECIST) version 1.1; (4) adequate organ functions; (5) the Eastern Cooperative Oncology Group performance status (ECOG) score of 0 or 1 ; (6) no central nervous system metastasis; (7) no prior treatment with liposomal doxorubicin or targeted drugs; (8) no history of known central nervous system metastases and prior malignant disease.

This study was approved by the Ethics Committee for Clinical Investigation of The Affiliated Cancer Hospital of Zhengzhou University, and carried out with ethics committee approval from each participating center. Before the start of treatment, because a patient was suffering from mental illness, the written informed consents were signed by his guardian, and all other patients signed their own written informed consent. The study followed all appropriate guidelines in accordance with the Helsinki Declaration.

\section{Treatment Protocol}

Patients were treated with daily oral anlotinib $12 \mathrm{mg} /$ day on days 1-14 and a maximum of six cycles of liposomal doxorubicin $50 \mathrm{mg} / \mathrm{m}^{2}$ on day 1 . Each cycle was 21 days, and the cycles were repeated every 21 days. Both drugs were continued until intolerable adverse events (AEs) or progressive disease (PD) occurred. After 1-6 cycles of liposomal doxorubicin in combination with anlotinib, if a complete response (CR), partial response (PR), or stable disease (SD) was observed, Anlotinib monotherapy was to be administered continuously until the detection of disease progression or appearance of unacceptable AEs. The study permitted two doses reduction levels anlotinib, from 
$12 \mathrm{mg} /$ day to $10 \mathrm{mg} /$ day and $8 \mathrm{mg} /$ day, and for liposomal doxorubicin from $50 \mathrm{mg} / \mathrm{m}^{2}$ to $42 \mathrm{mg} / \mathrm{m}^{2}$ and $36 \mathrm{mg} / \mathrm{m}^{2}$ depending on the AEs reported. If serious adverse events (SAEs) were observed, anlotinib and liposomal doxorubicin treatment were withheld until AEs reached grade1 or better, and the dose of both agents was reduced for all subsequent cycles.

\section{Assessment of Safety and Efficacy}

The tumor response assessments were performed at baseline, and every 6 weeks during the first six cycles, and then every 8 weeks until the detection of disease progression or appearance of unacceptable AEs. Response evaluation was performed with computed tomography (CT) or magnetic resonance imaging (MRI), and was divided into CR, PR, $\mathrm{SD}$, and PD according to RECIST v1.1. ORR was the proportion of patients who had CR and PR. DCR was the proportion of people obtained CR, PR, and SD. PFS was defined as the time from the initiation of the combined treatment to disease progression or death, whichever occurred first. AEs were collected and graded using National Cancer Institute Common Terminology Criteria for Adverse Events (NCI-CTC AE), version 4.0.

\section{Statistical Analysis}

All statistical data were analyzed by GraphPad Prism 8.11 software and SPSS 22.0 software. Quantitative variables were presented as medians (range) or numbers (percentage). The descriptive variables regarding patients' baseline characteristics and AEs were directly calculated from the database. PFS was plotted with Kaplan-Meier method, with $95 \%$ confidence interval (CI).

\section{Results}

A total of 27 patients were included in this study, including 11 males and 16 females. The median age of the patients was 45 years (range 20-63 years). Patient demographic and disease characteristics at baseline are summarized in Table 1. Primary tumors were distributed throughout the body, mainly in the extremities $(n=18)$. The site of metastasis was lung only in 23 patients, lymph node only in 2 patients and multiple organs in 2 patients. Histological subtypes were also significantly different. The most common subtypes were leiomyosarcoma $(n=8)$, followed by synovial sarcoma $(n=6)$, undifferentiated pleomorphic sarcoma $(\mathrm{n}=4)$, myxofibrosarcoma $(n=3)$, angiosarcoma $(n=2)$, epithelioid sarcoma $(n=2)$, rhabdomyosarcoma (RMS) $(\mathrm{n}=2)$. Twenty one $(77.8 \%)$ patients had received primary tumor resection, 13 (48.1\%) patients had failed to prior chemotherapy, and $11(40.7 \%)$ had prior radiation to the primary lesion.

Overall, the median number of liposomal doxorubicin cycles administered was 6 (range 1-6) and the median number of anlotinib cycles administered was 10 (range 1-20). During the combined treatment with anlotinib and liposomal doxorubicin, 8 patients discontinued treatment for disease progression $(n=7)$ and AEs $(n=1), 19$ patients continued anlotinib maintenance after the $1-6$ cycles of the combined treatment. At the cut-off time (15 September 2020), 3 patients remained on the treatment, while 24 patients had discontinued the treatment. The primary reason for treatment discontinuation was disease progression $(22 / 24,91.7 \%)$. Other reasons were AEs $(2 / 24,8.3 \%)$. In addition, $4(14.8 \%)$ patients needed one or more dose reductions. The median number of anlotinib maintenance cycles administered was 4 (range 1-14). AEs were collected for all patients.

\section{Efficacy}

All 27 patients were eligible for response evaluation. Two patients (one with synovial sarcoma and one with rhabdomyosarcoma) had CR and 9 patients obtained PR. The ORR was $40.7 \%$. Eleven patients experienced SD and 5 patients obtained SD (Table 2). The calculated DCR was $81.5 \%$ (Table 3 ). The average change in target lesion diameter from baseline is shown in Figure 1. The median follow-up was 8.9 months (range 2-15), the PFR at 3 and 6 months was $81.5 \%$ and $59.3 \%$, respectively (Table 3). The median PFS was 7 months $(95 \%$ CI, 5.3-8.1; Figure 2), and the median OS was not achieved at the time of analysis.

\section{Safety}

Table 4 lists the common treatment-related AEs throughout the treatment, including anlotinib combined with liposomal doxorubicin $(\mathrm{n}=27)$ and during anlotinib alone $(\mathrm{n}=19)$. The most common AEs were leukopenia (48.1\%), anemia (37\%), nausea/vomiting (33.3\%), fatigue (29.6\%), hypertension (29.6\%), elevated transaminase $(25.9 \%)$, and hand and foot skin reaction $(25.9 \%)$. The most frequent grade 3/4 AEs were leukopenia (33.3\%), febrile neutropenia (7.4\%), and anemia (7.4\%). During anlotinib-liposomal doxorubicin combined therapy, 10 (37\%) patients were observed with grade $3 / 4$ hematological AEs. During anlotinib alone, hypertension was the most common AEs, followed by hand and foot skin 
Table I Patient Demographics and Clinical Characteristics

\begin{tabular}{|c|c|c|}
\hline $\begin{array}{l}\text { Baseline } \\
\text { Characteristics }\end{array}$ & $\begin{array}{l}\text { Number of Patients } \\
\text { (n) }\end{array}$ & $\begin{array}{l}\text { Percentage } \\
\text { (\%) }\end{array}$ \\
\hline \multicolumn{3}{|l|}{ Age (years) } \\
\hline Median & 45 & \\
\hline Range & $20-63$ & \\
\hline \multicolumn{3}{|l|}{ Sex } \\
\hline Male & 11 & 40.7 \\
\hline Female & 16 & 59.3 \\
\hline \multicolumn{3}{|c|}{ ECOG performance status } \\
\hline 0 & 24 & 88.9 \\
\hline I & 2 & 7.4 \\
\hline 2 & I & 3.7 \\
\hline \multicolumn{3}{|l|}{ Histology } \\
\hline LMS & 8 & 29.6 \\
\hline SS & 6 & 22.2 \\
\hline UPS & 4 & 14.8 \\
\hline MFS & 3 & II.I \\
\hline AS & 2 & 7.4 \\
\hline ES & 2 & 7.4 \\
\hline RMS & 2 & 7.4 \\
\hline \multicolumn{3}{|l|}{ Primary tumor site } \\
\hline Extremities & 18 & 66.7 \\
\hline Trunk & 8 & 29.6 \\
\hline Head and neck & I & 3.7 \\
\hline \multicolumn{3}{|l|}{ Distant metastases } \\
\hline Lung & 23 & 85.2 \\
\hline Lymph node & 2 & 7.4 \\
\hline Multiple organs & 2 & 7.4 \\
\hline \multicolumn{3}{|c|}{ Radiotherapy history } \\
\hline Yes & 11 & 40.7 \\
\hline No & 16 & 59.3 \\
\hline \multicolumn{3}{|l|}{ Surgery history } \\
\hline Yes & 21 & 77.8 \\
\hline No & 6 & 22.2 \\
\hline \multicolumn{3}{|c|}{ Chemotherapy history } \\
\hline Yes & 13 & 48.1 \\
\hline No & 14 & 51.9 \\
\hline \multicolumn{3}{|c|}{ Chemotherapy drugs } \\
\hline $\mathrm{G}$ and $\mathrm{T}$ & 4 & 30.8 \\
\hline
\end{tabular}

(Continued)
Table I (Continued).

\begin{tabular}{|l|l|l|}
\hline $\begin{array}{l}\text { Baseline } \\
\text { Characteristics }\end{array}$ & $\begin{array}{l}\text { Number of Patients } \\
\text { (n) }\end{array}$ & $\begin{array}{l}\text { Percentage } \\
\text { (\%) }\end{array}$ \\
\hline Dox & 3 & 23.1 \\
Dox and IF & 4 & 30.8 \\
IF & 2 & 15.4 \\
\hline
\end{tabular}

Abbreviations: ECOG, Eastern Cooperative Oncology Group; LMS, leiomyosarcoma; SS, synovial sarcoma; UPS, undifferentiated pleomorphic sarcoma; MFS, myxofibrosarcoma; AS, angiosarcoma; ES, epithelioid sarcoma; RMS, rhabdomyosarcoma; G, gemcitabine; T, docetaxel; Dox, doxorubicin; IF, ifosfamide.

Table 2 Responses Data by Histologic Category

\begin{tabular}{|l|l|l|l|l|}
\hline \multirow{2}{*}{ Histological Subtypes } & \multicolumn{4}{|l|}{ Number of Patients } \\
\cline { 2 - 5 } & CR & PR & SD & PD \\
\hline Leiomyosarcoma & 0 & 3 & 4 & I \\
Synovial sarcoma & 1 & 2 & 2 & I \\
UPS & 0 & 1 & 2 & I \\
Myxofibrosarcoma & 0 & I & I & I \\
Angiosarcoma & 0 & 0 & I & I \\
Epithelioid sarcoma & 0 & 1 & I & 0 \\
Rhabdomyosarcoma & 1 & 1 & 0 & 0 \\
Total & 2 & 9 & II & 5 \\
\hline
\end{tabular}

Abbreviations: CR, complete response; PR, partial response; SD, stable disease; $\mathrm{PD}$, progressive disease; UPS, undifferentiated pleomorphic sarcoma.

reaction, proteinuria, leukopenia, and fatigue. These AEs were in line with those described in the drug description. No deaths related to the treatment were observed in this study.

Table 3 Tumor Responses ( $N=27)$

\begin{tabular}{|l|l|l|}
\hline \multirow{2}{*}{ Responses } & \multicolumn{2}{|l|}{ Anlotinib and Liposomal Doxorubicin } \\
\cline { 2 - 3 } & No & $\%$ \\
\hline CR & 2 & 7.4 \\
PR & 9 & 33.3 \\
SD & 11 & 40.7 \\
PD & 5 & 18.5 \\
ORR & 11 & 40.7 \\
DCR & 22 & 81.5 \\
Median PFS & 7.2 months & \\
PFR 3 months & & 81.5 \\
PFR 6 months & & 59.3 \\
\hline
\end{tabular}

Abbreviations: $\mathrm{CR}$, complete response; $\mathrm{PR}$, partial response; $\mathrm{SD}$, stable disease; $P D$, progressive disease; $O R R$, objective response rate; $D C R$, disease control rate; PFS, progression-free survival; PFR, progression-free rate. 


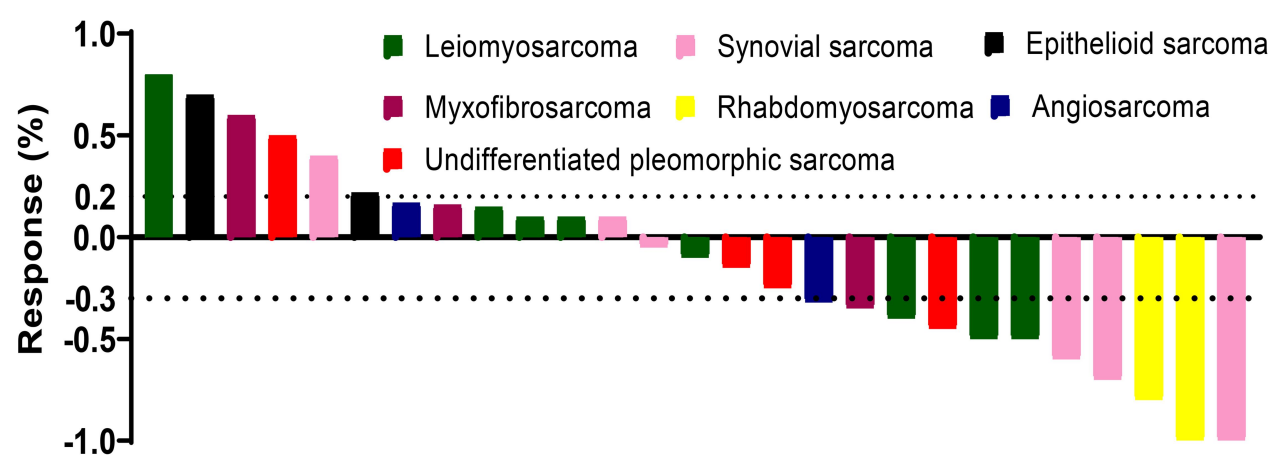

Patient

Figure I Waterfall plots for the maximum percentage change from baseline in size of target lesions during the treatment. The dashed lines represent the criteria for progressive disease ( $20 \%$ increase in target lesion size) and partial response ( $30 \%$ decrease in target lesion size) according to RECIST vl.I.

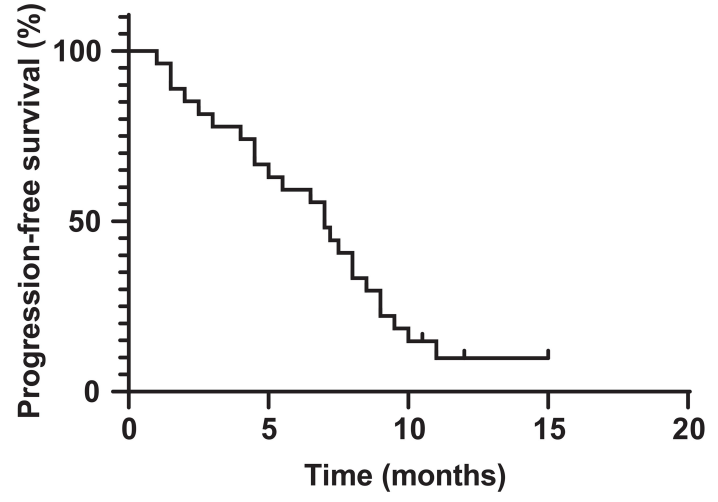

Figure 2 Kaplan-Meier curves of PFS.

\section{Discussion}

This study showed that anlotinib combined with liposomal doxorubicin followed by anlotinib maintenance was effective in patients with metastatic STS, the ORR was $40 \%$, DCR was $80 \%$, median PFS was 7 months $(95 \%$ CI, 5.3-8.1 months), and PFR at 3 and 6 months was $81.5 \%$ and $59.3 \%$, respectively. Although the dose was reduced in several patients, the combined therapy was well tolerated.

Doxorubicin is still first-line chemotherapy for most patients against advanced STS. Unfortunately, doxorubicin has the limitations of cumulative and irreversible myocardial toxicity and frequent neutropenic episodes, which are especially disadvantageous in the palliative setting. ${ }^{2}$ Liposomal doxorubicin showed decreased accumulation in tissues with tight junctions, such as the heart, and higher uptake by the fenestrated microvasculature of tumor tissue. ${ }^{14}$ Clinical studies showed that liposomal doxorubicin demonstrated equivalent antitumor activity and a favorable tolerability profile in terms of hematological, mucosal, and gastrointestinal toxicities, compared to doxorubicin. ${ }^{15}$ In a randomized Phase II trial, 94 patients with advanced or metastatic STS were enrolled. The response rates of patients treated with liposomal doxorubicin and doxorubicin were $10 \%$ and $9 \%$, respectively. The myelosuppressive and myocardial toxicity with liposomal doxorubicin were significantly less than that with doxorubicin. The main toxicity of liposomal doxorubicin was to the skin. ${ }^{8}$ A Phase II trial of 34 advanced STS patients showed that liposomal doxorubicin and ifosfamide

Table 4 Adverse Events

\begin{tabular}{|c|c|c|c|}
\hline Adverse Events & $\begin{array}{l}\text { Grade I-2 } \\
\text { No. (\%) }\end{array}$ & $\begin{array}{l}\text { Grade 3-4 } \\
\text { No. (\%) }\end{array}$ & $\begin{array}{l}\text { All Grade } \\
\text { No. (\%) }\end{array}$ \\
\hline Leukopenia & $4(14.8)$ & $9(33.3)$ & $13(48.1)$ \\
\hline Anemia & $8(29.6)$ & $2(7.4)$ & $10(37.0)$ \\
\hline Nausea/vomiting & $9(33.3)$ & $0(0)$ & $9(33.3)$ \\
\hline Hypertension & $8(29.6)$ & $0(0)$ & $8(29.6)$ \\
\hline Fatigue & $8(29.6)$ & $0(0)$ & $8(29.6)$ \\
\hline $\begin{array}{l}\text { Elevated } \\
\text { transaminases }\end{array}$ & $7(25.9)$ & $0(0)$ & $7(25.9)$ \\
\hline $\begin{array}{l}\text { Hand-foot skin } \\
\text { reaction }\end{array}$ & $6(22.2)$ & I (3.7) & 7 (25.9) \\
\hline Thrombocytopenia & $5(18.5)$ & I (3.7) & $6(22.2)$ \\
\hline Proteinuria & $5(18.5)$ & $0(0)$ & $5(18.5)$ \\
\hline $\begin{array}{l}\text { Febrile } \\
\text { neutropenia }\end{array}$ & $3(11.1)$ & $2(7.4)$ & $5(18.5)$ \\
\hline Alopecia & $4(14.8)$ & $0(0)$ & $4(14.8)$ \\
\hline Hypothyroidism & $4(14.8)$ & $0(0)$ & $4(14.8)$ \\
\hline $\begin{array}{l}\text { Triglyceride } \\
\text { elevation }\end{array}$ & $4(14.8)$ & $0(0)$ & $4(14.8)$ \\
\hline Anorexia & $3(11.1)$ & $0(0)$ & $3(11.1)$ \\
\hline Alopecia & $3(11.1)$ & $0(0)$ & $3(7.4)$ \\
\hline Diarrhea & $2(7.4)$ & $0(0)$ & $2(7.4)$ \\
\hline $\begin{array}{l}\text { Cholesterol } \\
\text { elevation }\end{array}$ & $2(7.4)$ & $0(0)$ & $2(7.4)$ \\
\hline Hoarse & $2(7.4)$ & $0(0)$ & $2(7.4)$ \\
\hline
\end{tabular}


achieved a modest activity, with an ORR of $55.9 \%$, a median PFS of 4.2 months, and a median OS of 11.2 months. ${ }^{15}$ Another Phase II trial showed that had modest efficacy as first-line treatment in patients with advanced STS, the median PFS was 5.7 months and median OS was 13.2 months, respectively, with an ORR of $16 \%{ }^{16}$ Liposomal doxorubicin also showed promising antitumor effect for patients who had failed to previous doxorubicin-based treatment. A Phase II trial conducted by the Italian Sarcoma Group showed liposomal doxorubicin obtained an ORR of $12 \%$ in 25 STS patients who were previously pretreated with doxorubicin-based chemotherapy, and only 2 patients experienced grade 3 toxicity. ${ }^{9}$ Over the past decade, preclinical studies showed that antiangiogenic agents could promote tumor vascular normalization and improve drug delivery and efficacy in many solid tumors, including STS. Bevacizumab combined with liposomal doxorubicin showed improved activity in advanced ovarian and Kaposi sarcoma. ${ }^{17}$ A Phase II trial showed that bevacizumab combined with liposomal doxorubicin had an active efficacy in patients with Kaposi sarcoma, with median PFS of 6.9 months and ORR of $56 \%{ }^{18}$ So, it may be reasonable to combine anlotinib with liposomal doxorubicin for better efficacy in the treatment of advanced STS.

This is the first report on the efficacy and safety of anlotinib combined with liposomal doxorubicin followed by anlotinib maintenance for advanced STS. In this study, OS was not achieved, and ORR, DCR and median PFS were chosen as the primary endpoints. CR occurred in 2 patients and PR was observed in 9 patients, the ORR was 40.7\%. Eleven patients obtained SD and 5 patients achieved PD, the DCR was $81.5 \%$. The median PFS was 7 months (95\% CI, 5.3-8.1 months). The PFR at 3 and 6 months was $81.5 \%$ and $59.3 \%$, respectively. Anlotinib and liposomal doxorubicin followed by anlotinib maintenance achieved high ORR and prolonged median PFS. This may be due to the synergistic effect that antiangiogenic drugs could reduce the generation of new blood vessels in tumors and promote the normalization of tumor blood vessels, which led to significantly increase the local drug concentration and enhance the antitumor activity of drugs. ${ }^{19,20}$ The result is consistent with previous reports. In a randomized Phase Ib/II trial, olaratumab combined with doxorubicin followed by olaratumab maintenance as the first-line therapy had an improvement in PFS (6.6 months vs 4.1 months) and OS (26.5 months vs 14.7 months) compared with doxorubicin alone in 133 patients with advanced STS. ${ }^{21}$ However, a large Phase III trial showed that no survival benefits were observed in similar patients who were treated with doxorubicin and olaratumab compared with doxorubicin alone. The different results of two trials may be due to the different trial designs and enrolled population. ${ }^{22}$ Another Phase II trial showed that pazopanib combined with gemcitabine, followed by pazopanib alone, as second-line treatment was beneficial for the patients with advanced leiomyosarcoma, but the study failed to meet its primary endpoint, the 9-month PFS was $32.1 \%$, with ORR of $23.8 \%$ and a median PFS of 6.5 months. The significant AEs were observed during pazopanib combined with gemcitabine, especially the hematological AEs. Most AEs were mild during maintenance pazopanib. ${ }^{23}$ Other studies reported the limited efficacy of antiangiogenic drugs combined chemotherapy in the treatment of advanced STS. In a nonrandomized Phase II trial, 17 patients with metastatic STS received doxorubicin combined with bevacizumab during the first stage of the study, only 5 of whom received maintenance therapy with bevacizumab. The ORR of $12 \%$ was so low that recruitment was stopped. ${ }^{24}$

When patients are treated with a potentially effective combination of drugs, it is critical to consider combined treatment AEs. In this study, anlotinib $12 \mathrm{mg}$ /day on days 1-14 and liposomal doxorubicin $50 \mathrm{mg} / \mathrm{m} 2$ on day 1 every 21 days did not significantly increased frequency and severity of adverse reactions. Most AEs were mild and acceptable, the most common AEs were leukopenia (48.1\%), anemia (37\%), nausea/vomiting (33.3\%), fatigue (29.6\%), hypertension (29.6\%), elevated transaminase (25.9\%), and hand and foot skin reaction (25.9\%). The most common grade 3/4 AEs were leukopenia (33.3\%), anemia $(7.4 \%)$, febrile neutropenia $(7.4 \%)$. Overall, the AEs were mainly reported during the combined treatment with anlotinib and liposomal doxorubicin, especially, grade 3/4 hematological AEs. Most of them were reversible with appropriate medical management or dose reduction. Few grades 3/4 AEs were observed during anlotinib alone. In view of the tolerable AEs of this combination therapy with anlotinib and liposomal doxorubicin, it is necessary to further explore the value of the combined treatment in prospective studies of advanced STS.

Maintenance therapy aims to maintain tumor response, alleviate symptoms, and improve quality of life. It has been proven to be effective in a variety of malignant tumors, including lung cancer and STS. In a retrospective study included 885 patients after failure 
or intolerance to doxorubicin and ifosfamide, after six cycles of trabectedin treatment, patients with nonprogressive disease treated with trabectedin until disease progression had a better median PFS (11.7 months vs 7.6 months, $\mathrm{P}<0.003)$ and median OS $(24.9$ months vs 16.9 months, $\mathrm{P}<0.001)$ than those patients who stopped trabectedin treatment. $^{25}$ There is one ongoing trial of TKI maintenance in STS, the study is to assess the efficacy of regorafenib compared to placebo as maintenance therapy in patients with metastatic STS who experienced stable disease or response after 6 cycles of doxorubicin-based chemotherapy (NCT03793361). ${ }^{26}$ The treatment would be administrated as long as it appears beneficial. In our study, anlotinib maintenance may be feasible and well tolerated.

There are some limitations in this study. First of all, because of heterogeneity of sarcomas, the sample size and retrospective nature of this study, we could not draw a definite conclusion and analyze the difference of efficacy between chemonaive and previously treated patients. In addition, the optimal dose of liposomal doxorubicin and anlotinib needs to be further investigated.

\section{Conclusion}

In summary, anlotinib combined with liposomal doxorubicin followed by anlotinib maintenance is effective in patients with metastatic STS, and the AEs profile encountered is mild and acceptable. Further prospective randomized controlled trials are needed to confirm the advantages of this combined therapy.

\section{Ethical Statement}

This study was approved by the Ethics Committee for Clinical Investigation of The Affiliated Cancer Hospital of Zhengzhou University, and carried out with ethics committee approval from each participating center. All procedures performed in studies involving human participants were in accordance with the ethical standards of the institutional and/or national research committee and with the 1964 Helsinki declaration and its later amendments or comparable ethical standards. Written informed consent was obtained from all patients or their guardians before the therapy procedures.

\section{Author Contributions}

All authors made substantial contributions to conception and design, acquisition of data, or analysis and interpretation of data; took part in drafting the article or revising it critically for important intellectual content; agreed to submit to the current journal; gave final approval of the version to be published; and agree to be accountable for all aspects of the work.

\section{Disclosure}

The authors report no conflicts of interest in this work.

\section{References}

1. Ferrari A, Sultan I, Huang TT, et al. Soft tissue sarcoma across the age spectrum: a population-based study from the surveillance epidemiology and end results database. Pediatr Blood Cancer. 2011;57 (6):943-949. doi:10.1002/pbc. 23252

2. Judson I, Verweij J, Gelderblom H, et al. Doxorubicin alone versus intensified doxorubicin plus ifosfamide for first-line treatment of advanced or metastatic soft-tissue sarcoma: a randomised controlled phase 3 trial. Lancet Oncol. 2014;15(4):415-423. doi:10.1016/S14702045(14)70063-4

3. Nakano K, Takahashi S. Precision medicine in soft tissue sarcoma treatment. Cancers. 2020;12(1):221. doi:10.3390/cancers12010221

4. Maki RG, Wathen JK, Patel SR, et al. Randomized phase II study of gemcitabine and docetaxel compared with gemcitabine alone in patients with metastatic soft tissue sarcomas: results of sarcoma alliance for research through collaboration study 002 [corrected]. J Clin Oncol. 2007;25(19):2755-2763. doi:10.1200/ JCO.2006.10.4117

5. Rosen G, Forscher C, Lowenbraun S, et al. Synovial sarcoma. Uniform response of metastases to high dose ifosfamide. Cancer. 1994;73 (10):2506-2511. doi:10.1002/1097-0142(19940515)73:10<2506::AIDCNCR2820731009>3.0.CO;2-S

6. Penel N, Bui BN, Bay J-O, et al. Phase II trial of weekly paclitaxel for unresectable angiosarcoma: the ANGIOTAX Study. J Clin Oncol. 2008;26(32):5269-5274. doi:10.1200/JCO.2008.17.3146

7. Kobayashi H, Iwata S, Wakamatsu T, et al. Efficacy and safety of trabectedin for patients with unresectable and relapsed soft-tissue sarcoma in Japan: A Japanese Musculoskeletal Oncology Group study. Cancer. 2020;126(6):1253-1263. doi:10.1002/cncr.32661

8. Judson I, Radford JA, Harris M, et al. Randomised phase II trial of pegylated liposomal doxorubicin (DOXIL/CAELYX) versus doxorubicin in the treatment of advanced or metastatic soft tissue sarcoma: a study by the EORTC Soft Tissue and Bone Sarcoma Group. Eur $J$ Cancer. 2001;37(7):870-877. doi:10.1016/S0959-8049(01)00050-8

9. Toma S, Tucci A, Villani G, Carteni G, Spadini N, Palumbo R. Liposomal doxorubicin (Caelyx) in advanced pretreated soft tissue sarcomas: a phase II study of the Italian Sarcoma Group (ISG). Anticancer Res. 2000;20(1B):485-491.

10. Sun Y, Niu W, Du F, et al. Safety, pharmacokinetics, and antitumor properties of anlotinib, an oral multi-target tyrosine kinase inhibitor, in patients with advanced refractory solid tumors. J Hematol Oncol. 2016;9(1):105. doi:10.1186/s13045-016-0332-8

11. Chi Y, Fang Z, Hong X, et al. Safety and efficacy of anlotinib, a multikinase angiogenesis inhibitor, in patients with refractory metastatic soft-tissue sarcoma. Clin Cancer Res. 2018;24(21):5233-5238. doi:10.1158/1078-0432.CCR-17-3766

12. Chi Y, Yao Y, Wang S, et al. Anlotinib for metastasis soft tissue sarcoma: a randomized, double-blind, placebo-controlled and multi-centered clinical trial. $J$ Clin Oncol. 2018;36 (15_suppl):11503. doi:10.1200/JCO.2018.36.15_suppl.11503

13. Shi J, Zhang Y, Wang J, Li J, Li Z. Anlotinib combined with chemoradiotherapy exhibits significant therapeutic efficacy in esophageal squamous cell carcinoma. Front Oncol. 2020;10:995. doi: $10.3389 /$ fonc 2020.00995 
14. Allen TM, Martin FJ. Advantages of liposomal delivery systems for anthracyclines. Semin Oncol. 2004;31(6 Suppl 13):5-15. doi:10.1053/j.seminoncol.2004.08.001

15. De Sanctis R, Bertuzzi A, Basso U, et al. Non-pegylated liposomal doxorubicin plus ifosfamide in metastatic soft tissue sarcoma: results from a phase-II trial. Anticancer Res. 2015;35(1):543-547.

16. Bafaloukos D, Papadimitriou C, Linardou H, et al. Combination of pegylated liposomal doxorubicin (PLD) and paclitaxel in patients with advanced soft tissue sarcoma: a phase II study of the Hellenic Cooperative Oncology Group. Br J Cancer. 2004;91(9):1639-1644. doi:10.1038/sj.bjc.6602148

17. Verschraegen CF, Czok S, Muller CY, et al. Phase II study of bevacizumab with liposomal doxorubicin for patients with platinum- and taxane-resistant ovarian cancer. Ann Oncol. 2012;23 (12):3104-3110. doi:10.1093/annonc/mds172

18. Ramaswami R, Uldrick TS, Polizzotto MN, et al. A pilot study of liposomal doxorubicin combined with bevacizumab followed by bevacizumab monotherapy in patients with advanced kaposi sarcoma. Clin Cancer Res. 2019;25(14):4238-4247. doi:10.1158/1078-0432. CCR-18-3528

19. Tolaney SM, Boucher Y, Duda DG, et al. Role of vascular density and normalization in response to neoadjuvant bevacizumab and chemotherapy in breast cancer patients. Proc Natl Acad Sci USA. 2015;112(46):14325-14330. doi:10.1073/pnas.1518808112

20. Sorensen AG, Batchelor TT, Zhang W-T, et al. A "vascular normalization index" as potential mechanistic biomarker to predict survival after a single dose of cediranib in recurrent glioblastoma patients. Cancer Res. 2009;69(13):5296-5300. doi:10.1158/0008-5472.CAN09-0814
21. Tap WD, Jones RL, Van Tine BA, et al. Olaratumab and doxorubicin versus doxorubicin alone for treatment of soft-tissue sarcoma: an open-label phase $1 \mathrm{~b}$ and randomised Phase 2 trial. Lancet. 2016;388(10043):488-497. doi:10.1016/S0140-6736(16)30587-6

22. Tap WD, Wagner AJ, Schöffski P, et al. Effect of doxorubicin plus olaratumab vs doxorubicin plus placebo on survival in patients with advanced soft tissue sarcomas: the ANNOUNCE randomized clinical trial. JAMA. 2020;323(13):1266-1276. doi:10.1001/jama.2020.1707

23. Pautier P, Penel N, Ray-Coquard I, et al. A phase II of gemcitabine combined with pazopanib followed by pazopanib maintenance, as second-line treatment in patients with advanced leiomyosarcomas: a unicancer French Sarcoma Group study (LMS03 study). Eur J Cancer. 2020;125:31-37. doi:10.1016/j.ejca.2019.10.028

24. D'Adamo DR, Anderson SE, Albritton K, et al. Phase II study of doxorubicin and bevacizumab for patients with metastatic soft-tissue sarcomas. J Clin Oncol. 2005;23(28):7135-7142. doi:10.1200/ JCO.2005.16.139

25. Le Cesne A, Ray-Coquard I, Duffaud F, et al. Trabectedin in patients with advanced soft tissue sarcoma: a retrospective national analysis of the French Sarcoma Group. Eur J Cancer. 2015;51(6):742-750. doi:10.1016/j.ejca.2015.01.006

26. Centre Oscar Lambret. Phase II study of regorafenib as maintenance therapy. NLM identifier: NCT03793361. Available from: https://clin icaltrials.gov/ct2/show/study/NCT03793361. Accessed December 1, 2020.

\section{Publish your work in this journal}

Cancer Management and Research is an international, peer-reviewed open access journal focusing on cancer research and the optimal use of preventative and integrated treatment interventions to achieve improved outcomes, enhanced survival and quality of life for the cancer patient.
The manuscript management system is completely online and includes a very quick and fair peer-review system, which is all easy to use. Visit http://www.dovepress.com/testimonials.php to read real quotes from published authors. 